\title{
CHEMOBRAIN: REVISIÓN DE ESTUDIOS QUE EVALÚAN EL DETERIORO COGNITIVO DE SUPERVIVIENTES DE CÁNCER TRATADOS CON QUIMIOTERAPIA
}

\section{CHEMOBRAIN: REVIEW OF STUDIES ASSESSING COGNITIVE IMPAIRMENT IN CANCER SURVIVORS TREATED WITH CHEMOTHERAPY}

\author{
Sonia López-Santiago', Juan A. Cruzado' y Jaime Feliú2 \\ ' Facultad de Psicología. Universidad Complutense de Madrid \\ 2 Servicio de Oncología Médica. Hospital Universitario la Paz de Madrid
}

Resumen

Planteamiento del problema: Algunos pacientes oncológicos han expresado problemas de atención, concentración y memoria durante y después de los tratamientos con quimioterapia, a los que se ha llamado chemobrain. Como consecuencia de estas manifestaciones, en las últimas décadas los estudios dedicados a indagar este posible efecto adverso se han incrementado.

Desarrollo del tema: Este trabajo pretende revisar y analizar los estudios científicos más recientes que investigan la naturaleza y alcance del daño cognitivo asociado a los tratamientos quimioterapéuticos. Los artículos analizados se han extraído de diferentes fuentes bibliográficas, principalmente de la base de datos PubMed. Como resultado de la búsqueda se aprecia que, en los últimos años, se han incremento los estudios de diseño longitudinal; este posible efecto adverso se sigue estudiando sobre todo en muestras de mujeres con cáncer de mama, las investigaciones en otros tipos de cánceres son insuficientes; así mismo, destaca la inclusión de nuevas herramientas de evaluación, como la resonancia magnética y el electroencefalograma, junto con nuevos experimentos realizados con animales, in vivo e in vitro, finalmente se constata la inclusión de algunos estudios sobre fármacos y estrategias eficaces para su afrontamiento. La elaboración de guías

\section{Abstract}

The problem: Some cancer patients have expressed attention, concentration and memory problems, during and after their treatment with chemotherapy, also called chemobrain. Following these complains, in recent decades, studies about this adverse effect had increased.

Development of the topic: this work aims to review and analyze the latest scientific studies investigating the nature and severity of cognitive impairment associated with chemotherapy treatments. Works discussed are drowned from different bibliographical sources, mainly from the PubMed database. Results show that, in recent years, longitudinal studies have increased, chemobrain is being studied above all in samples from women with breast cancer, research in other types of cancer is insufficient. Also, the works include new assessment tools, such as MRI and EEG. Advances are done with animal experiments, in vivo and in vitro. Finally, we found studies on drugs and effective strategies for coping of cognitive impairment. The development of guidelines for its systematic study and recommendations for its approach are still pending.

Conclusion: There is not conclusive data on the chemobrain, longitudinal studies should be encouraged, as well as assessing the cogniti-

\section{Correspondencia:}


para el estudio sistemático y las recomendaciones para su abordaje aún quedan pendientes.

Conclusiones: no existen datos concluyentes sobre el chemobrain, los estudios longitudinales deberán incentivarse, así como la valoración del impacto cognitivo de determinados esquemas quimioterapéuticos en diferentes cánceres. Los trabajos sobre el afrontamiento, psicológico y/o farmacológico, de este problema deben continuar.

Palabras clave: Quimioterapia, cáncer, deterioro cognitivo, chemobrain.

\section{INTRODUCCIÓN}

En las últimas décadas, el estudio del cáncer y sus tratamientos está ocupando un lugar destacado en el ámbito de la investigación. Los estudios del genoma humano profundizan en el conocimiento de las mutaciones genéticas específicas para determinados tipos de cáncer. El estudio biológico permite conocer el comportamiento de las células oncológicas. Los avances en estos estudios facilitan la creación de tratamientos más específicos y eficaces, que quedan patentes en un evidente y alentador incremento de la supervivencia de las personas afectadas.

Sin embargo, la realidad actual de los enfermos y enfermas oncológicos sigue siendo compleja. La enfermedad no sólo provoca un impacto físico, sino que, además, pueden acontecer daños bio-psicosociales colaterales a la propia enfermedad y a los tratamientos antineoplásicos. Conscientes de esta circunstancia, los profesionales de la oncología y la salud tienen presentes los efectos secundarios de las terapias aplicadas, con el fin de controlarlos y permitir una mejor asimilación del tratamiento por parte del paciente. Por tanto, es especialmente relevante conocer los efectos adversos de los tratamientos. Este conocimiento puede conducir a una mejor prevención y contención de los mismos $y$, por ende, procurar un mayor bienestar ve impact of certain chemotherapeutic agents in different cancers. The works on cognitive impairment's coping, psychological and drug, should continue.

Keywords: Chemotherapy, cancer, cognitive impairment, chemobrain. y mejor calidad de vida del paciente que los sufre.

Existe un conjunto de tratamientos destacados en la lucha contra el cáncer, la cirugía, la radioterapia, la hormonoterapia, la quimioterapia. Por sus múltiples aplicaciones, la quimioterapia es uno de los más importantes, pudiendo estar indicada en el tratamiento neoadyuvante, adyuvante o paliativo del cáncer. En nuestro país la reciben a diario miles de personas. De ahí que el desarrollo de los esquemas quimioterapéuticos esté en continua evolución y mejora, principalmente orientándose hacia el logro de una mayor especificidad (una respuesta terapéutica más eficiente mediante el ataque selectivo a las células cancerosas y una mayor conservación de las células normales). Muchos de sus efectos secundarios son bien conocidos mientras otros están bajo el punto de mira de importantes investigaciones internacionales y nacionales. Este es el caso de la valoración de un posible deterioro cognitivo asociado a la administración de agentes quimioterapéuticos.

En la práctica clínica, algunos pacientes manifiestan problemas de concentración y memoria durante y después del tratamiento con quimioterapia. Desafortunadamente, en nuestro país, estas quejas pasan con frecuencia desapercibidas. Los trabajos de investigación al respecto aún son insuficientes y por ello las tareas de prevención 
y control de este posible efecto secundario son tareas pendientes. No es hasta 2009, cuando en nuestro país se publican dos trabajos acerca de esta problemática en una revista especializada en psicooncología. Rubio, Sirgo, Forcadell, Mele y Guma, quienes realizan una revisión de los estudios científicos que indagan el deterioro cognitivo inducido por los tratamientos oncológicos sistémicos en el cáncer de mama no metastático ${ }^{(1)}$. Y la publicación de López, Cruzado y Feliú(2) un estudio que arroja resultados preliminares hallados en rendimiento cognitivo, estado emocional y calidad de vida en pacientes de cáncer de colon previamente a recibir tratamiento con quimioterapia.

La finalidad de este trabajo se centra en valorar el estado científico actual respecto al estudio del daño cognitivo quimio-inducido y analizar las líneas de investigación actuales.

\section{EL ESTUDIO DE UN FENÓMENO LLAMADO "CHEMOBRAIN"}

Algunos pacientes oncológicos manifiestan dificultades de concentración y memoria durante el proceso de curación de la enfermedad. A estos problemas cognitivos se les viene llamando coloquialmente "chemofog" o "chemobrain", términos que reflejan cómo los pacientes atribuyen estas dificultades a su tratamiento con quimioterapia.

A raíz de las quejas de los pacientes se ha desarrollado un creciente cuerpo de investigación interesado en indagar la naturaleza y mecanismos de este posible efecto adverso del tratamiento quimioterapéutico. Los datos ponen de manifiesto que un subgrupo de pacientes puede sufrir deterioro cognitivo quimio-inducido, aunque en muchos de estos casos el daño podría ser sutil. No obstante, incluso un pequeño deterioro puede tener una importante repercusión, pues las tareas cotidia- nas de la vida, desde las más sencillas a las más complejas, tanto las que requieren un control voluntario como las automatizadas, exigen un correcto funcionamiento de los dominios o funciones cognitivas. Cualquier alteración cerebral, de carácter estructural o funcional, puede producir un deterioro neuropsicológico que, a su vez, puede comprometer la capacidad adaptativa de la persona.

Según Vardy ${ }^{(3)}$, los estudios señalan que entre el 15 y el $50 \%$ de los pacientes que reciben quimioterapia podría experimentar este efecto secundario, entendido como problemas en memoria, aprendizaje, concentración, razonamiento, función ejecutiva, atención y habilidad visoespacial, durante y después de la quimioterapia ${ }^{(4)}$.

Se postulan varías vías por las que el tratamiento sistémico podría producir tales efectos adversos ${ }^{(5)}$, por vía directa mediante un efecto neurotóxico (considerando cierta permeabilidad de la barrera hematoencefálica a los agentes químicos empleados en el tratamiento sistémico) e indirecta modificando otras respuestas biológicas (por ejemplo modulando la respuesta inmune o provocando cambios hormonales $\left.{ }^{(3)}\right)$.

Sin embargo, existen datos que han revelado daños cognitivos previos al tratamiento, que oscilan entre el 20 y el $40 \%{ }^{(6,-8)}$. El deterioro previo, junto con la influencia de otras variables personales (como la edad, el nivel de estudios, los estados emocionales alterados), circunstanciales (como el tipo de instrumento de evaluación seleccionado) y físicas (como la comorbilidad, el estadio del cáncer, la presencia de anemia) hacen pensar que la realidad del Ilamado chemobrain es mucho más compleja ${ }^{(9)}$.

\section{LÍNEAS DE INVESTIGACIÓN ACTUALES}

La falta de consenso existente sobre la naturaleza y el alcance del daño cognitivo asociado a la quimioterapia sigue moti- 
vando la realización de múltiples trabajos científicos. En los últimos cinco años, se observa un incremento de los estudios longitudinales, un interés por ampliar el estudio a otros tipos de cáncer, la incorporación de estudios con animales y el uso de nuevas herramientas de evaluación, el esfuerzo por determinar los mecanismos subyacentes al deterioro cognitivo, la evaluación de la eficacia de propuestas de tratamiento, y los continuos esfuerzos por mejorar la metodología. Veamos estas líneas de investigación con mayor detenimiento.

\section{ESTUDIOS LONGITUDINALES}

En las primeras décadas del estudio de las alteraciones cognitivas asociadas al tratamiento sistémico, se pusieron en marcha trabajos de investigación de diseño transversal, que pusieron de manifiesto la existencia de este efecto adverso. Pese a tener en cuenta su importante aportación, no estuvieron exentos de críticas metodológicas y mostraron una importante desventaja: la dificultad de realizar una atribución causal correcta. Al carecer de línea base previa a la aplicación de quimioterapia, estos trabajos ignoraban la posible existencia de deterioro cognitivo anterior a la misma. Por tanto, las alteraciones cognitivas encontradas eran erróneamente atribuidas al tratamiento, realizando en muchas ocasiones una sobreestimación del daño quimio-inducido. Las recomendaciones de dichos trabajos alentaban la realización de investigaciones de corte longitudinal. No obstante, los estudios prospectivos requieren un mayor esfuerzo exigido por parte de los científicos y las organizaciones, debido a una mayor necesidad de recursos humanos, materiales y técnicos.

Muchos de estos nuevos trabajos cuentan para su realización con la participación de mujeres con cáncer de mama. A continuación se exponen brevemente al- gunos de resultados obtenidos por estos estudios:

- La quimioterapia adyuvante puede producir deterioro cognitivo persistente en el tiempo en un subgrupo de pacientes ${ }^{(8,11)}$, mientras que en otros casos puede haber una tendencia a la recuperación con el paso del tiempo ${ }^{(12-14)}$.

- Algunos dominios cognitivos pueden tener mayor propensión a sufrir deterioro (ver Tabla 1$)^{(8,13-15)}$

- El déficit puede mantener una relación dosis-dependiente, presentando mayor riesgo las mujeres que reciben quimioterapia a dosis altas ${ }^{(16)}$.

- La combinación de tratamientos antineoplásicos, por ejemplo quimioterapia más tamoxifeno, puede provocar alteraciones cognitivas más severas ${ }^{(10)}$. En monoterapia, el tratamiento quimioterapéutico produce más deterioro que el hormonal $(31 \text { vs. } 12 \%)^{(17)}$; frente a la radioterapia la quimioterapia produce daños específicos en fluencia verbal, aunque ambos dificulten la memoria verbal ${ }^{(18)}$.

- Existe escasa concordancia entre los test neuropsicológicos y la percepción subjetiva del daño(19). Ambos tipos de datos ofrecen información relevante. Un trabajo evaluó la percepción de 45 mujeres mayores con cáncer de mama (edad media 70 años) y apreciaron que la mitad de ellas consideraban que sus capacidades habían disminuido, sobre todo la capacidad de aprender información nueva (49\%), seguida de memoria de trabajo (29\%) y recuerdo remoto $(20 \%)^{(20)}$; otro estudio encuentra un elevado porcentaje de quejas cognitivas en memoria y concentración, aún presentes tras 18 meses del tratamiento, por lo que cuestionan la sensibilidad de las pruebas objetivas a sutiles déficit que pueden alterar la vida del paciente ${ }^{(21)}$.

- El rendimiento objetivo en las pruebas no se relacionó con otras variables como malestar psicológico, calidad de vida y fatiga ${ }^{(22)}$. 


\section{Tabla 1. Dominios cognitivos afectados en mujeres con cáncer de mama tratadas con quimioterapia}

\begin{tabular}{|l|l|}
\hline \multicolumn{1}{|c|}{ Estudio (año de publicación) } & \multicolumn{1}{|c|}{ Dominios cognitivos } \\
\hline $\begin{array}{l}\text { Wagner, Sweet, Butt, Beaumont, Havlin, } \\
\text { Sabatino et al. }(2006)^{(12)}\end{array}$ & $\begin{array}{l}\text { Atención, habilidad viso-espacial y me- } \\
\text { moria de trabajo }\end{array}$ \\
\hline $\begin{array}{l}\text { Jansen, Dodd, Miaskowski, Dowling y } \\
\text { Kramer (2008) })^{(13)}\end{array}$ & $\begin{array}{l}\text { Habilidad viso-espacial y en puntuacio- } \\
\text { nes totales cognitivas }\end{array}$ \\
\hline $\begin{array}{l}\text { Ahles, Saykin McDonald, Li, Furstenberg, } \\
\text { Hanscom et al. }(2010)^{(14)}\end{array}$ & $\begin{array}{l}\text { Habilidad verbal, daño a corto plazo me- } \\
\text { diado por la edad y la reserva cognitiva. }\end{array}$ \\
\hline Wefel, Saleeba, Buzdar y Meyers $(2010)^{(8)}$ & $\begin{array}{l}\text { Aprendizaje, memoria, función ejecutiva } \\
\text { y velocidad de procesamiento }\end{array}$ \\
\hline
\end{tabular}

Algunos trabajos no han encontrado evidencia de déficit cognitivo tras la aplicación de quimioterapia, pero estos son minoritarios: en su estudio, con 19 mujeres con cáncer de mama temprano, detectan un deterioro cognitivo previo a la aplicación de quimioterapia pero no se perciben daños asociados al tratamiento, el tamaño de la muestra hace que estos resultados sean discutibles ${ }^{(6)}$. Con una muestra algo mayor de enfermas de cáncer de mama ( $n=34)$ en comparación con pacientes cardiacos $(\mathrm{n}=12)$ y controles sanos ( $\mathrm{n}$ = 12), no corroboran la hipótesis de un daño cognitivo asociado a dosis estándar de quimioterapia ${ }^{(23)}$. Mientras otro trabajo sólo encontró enlentecimiento motor en 61 mujeres mayores con cáncer de mama no metastático, este deterioro podría estar asociado a neuropatía periférica ${ }^{(24)}$.

\section{Estudios realizados en poblaciones con diferentes tipos de cáncer}

Siguen siendo escasos los trabajos que se ocupan de explorar los problemas cognitivos asociados a la terapia sistémica en otros tipos de cánceres, apareciendo estudios transversales y longitudinales en los últimos años. Destacamos algunos de los resultados obtenidos:
- Cáncer hematológico y del tracto intestinal: en 2006, Eberhardt, Dilger, Musial, Wedding, Weissn y Miltner publican varios trabajos al respecto: en una muestra de 130 participantes, al comparar los pacientes no tratados frente a aquellos que habían recibido quimioterapia hacía pocos días, existía un peor rendimiento en la fluencia verbal, el aprendizaje verbal y la memoria en quienes habían recibido el tratamiento; la edad fue una variable relacionada con el deterioro en memoria ${ }^{(25)}$. En una muestra de 77 pacientes, observaron que a los seis meses del inicio del tratamiento hubo una mejoría en aprendizaje verbal, fluencia verbal y memoria, siendo la recuperación independiente de la edad de los participantes ${ }^{(26)}$.

- Cáncer de pulmón: la anemia derivada de la quimioterapia podría inducir alteraciones en la funcionalidad y rendimiento cognitivo; por lo tanto, el tratamiento de la anemía podría mejorar ambos ${ }^{(27)}$.

- Cáncer de colon: Vardy, Rourke, Pond, Galica, Park, Dhillon et al., aprecian un rendimiento cognitivo peor en pacientes de cáncer de colon antes de ser tratados con quimioterapia y una tendencia a empeorar a los doce meses de haberles administrado el tratamiento $(n=127)$ frente a los pacientes no tratados $(n=55)$, no 
hallando diferencias entre ambos grupos a los seis meses del final de la terapia ${ }^{(28)}$. Otro estudio realizado por los mismos autores en una amplia muestra de pacientes de cáncer de mama y de colon (302), destaca que las quejas cognitivas se asocian a malestar psicológico, fatiga, calidad de vida y administración de quimioterapia, pero estas quejas no coinciden con los resultados de las pruebas neuropsicológicas; resaltan la importancia de aplicar tanto instrumentos de percepción subjetiva del funcionamiento cognitivo como test objetivos $^{(29)}$. En una muestra de 327 pacientes de cáncer de colon se observó que el 10\% ponían de manifiesto quejas cognitivas, y un $36 \%$ mostró deterioro cognitivo en test neuropsicológicos clásicos. Los pacientes con un cáncer más avanzado, estadio IV, se sentían más fatigados y obtuvieron puntuaciones mayores en depresión y ansiedad $^{(30)}$. Datos obtenidos por nuestro equipo de investigación, corroboran un deterioro cognitivo, en función ejecutiva, memoria verbal y habilidad psicomotora, previo a la aplicación de quimioterapia en 35 pacientes, el deterioro se asoció a la edad, el nivel educativo bajo, problemas físicos y psico-sociales ${ }^{(2)}$.

- Cáncer testicular: al comparar a 70 pacientes tratados con cirugía+quimioterapia frente a 57 tratados con cirugía+radioterapia y 55 con cirugía sola, observaron que los participantes presentaban con frecuencia quejas cognitivas, independientemente de la modalidad de tratamiento, pero que solo un pequeño grupo de los tratados con quimioterapia manifestaba deterioro en pruebas neuropsicológi$\operatorname{cas}^{(31)}$. En una muestra de 69 pacientes, evaluados tras la cirugía y antes de recibir quimioterapia, apreciaron un $43 \%$ de deterioro cognitivo, el deterioro fue evidente en función motora, aprendizaje verbal y función ejecutiva ${ }^{(32)}$.

- Cáncer de ovario: en el estudio de 638 mujeres que recibieron quimioterapia, y otras 68 que no la habían recibido, se observa que las mujeres que habían recibido este tratamiento presentaban más quejas en memoria. Las quejas estaban asociadas a fatiga, cambios de humor, sofocos o escalofríos y problemas de sueño ${ }^{(33)}$.

\section{Estudios con animales}

Una interesante línea de investigación que se ha potenciado en los últimos años son los estudios con animales. Mediante estos trabajos se consigue realizar experimentos que, de otra forma, no serían éticamente factibles en humanos. Además, arrojan nuevos datos sobre la influencia de la quimioterapia en la capacidad cognitiva de sujetos experimentales sanos o enfermos, permitiendo un control más exhaustivo de variables independientes. Analizan la correspondencia con los daños cerebrales y cognitivos asociados a la quimioterapia ocasionados en humanos ${ }^{(34)}$ y sobrepasan el obstáculo de evaluar la neurotoxicidad de estos fármacos sin el influjo de la enfermedad de cáncer, surgiendo constantemente aportaciones nuevas.

Un trabajo comparativo de varios fármacos (ciclofosfamida y 5 -fluororacilo), que fueron aplicados a ratas jóvenes y adultas, mostró un efecto tóxico más evidente con la ciclofosfamida; pese a dicha toxicidad, se produjo una mejoría transitoria en el rendimiento en dos tareas de aprendizaje espacial y memoria. Como posibles hipótesis se plantean: que la susceptibilidad a la neurotoxicidad esté influida por el estadio del tumor, proponiendo realizar estudios en animales enfermos; que una restricción en la dieta de estos animales pueda ejercer un efecto neuroprotector ante neurotóxicos; o bien que las tareas de evaluación empleadas no sean sensibles a los dominios cognitivos alterados en pacientes tratados con quimioterapia ${ }^{(35)}$.

Otros muchos trabajos de este tipo sí ratifican un daño en tareas que implican 
procesamiento cognitivo. En el trabajo de Reiriz, Reolon, Preissles, Rosado, Henriques, Roesler et al., aparece un deterioro transitorio en la memoria de los ratones tras el empleo de un modelo de condicionamiento aversivo que requiere el correcto funcionamiento del hipocampo ${ }^{(36)}$. Otros experimentos permiten comprender el mecanismo por el que un fármaco, incapaz de atravesar la barrera hematoencefálica, puede producir pérdidas de memoria y deterioro en la capacidad de realizar tareas complejas; en concreto la adriamicina podría favorecer el vertido de citoquinas al torrente sanguíneo y contar con el óxido nítrico como un importante mediador ${ }^{(37)}$. Frente a las inyecciones placebo, administrar adriamicina o ciclofosfamida provoca alteración de la memoria en ratas de laboratorio sin enfermedad ${ }^{(38)}$. Los datos electrofisiológicos sugieren deterioro en la percepción de imputs auditivos como posible factor etiológico del daño $\operatorname{cognitivo}{ }^{(39)}$. La combinación de agentes quimioterapéuti$\mathrm{cos}$ (como 5-FU+metotrexato) produce mayor dificultad de recuperar comportamientos ya aprendidos que cuando se emplean en monoterapia ${ }^{(40)}$. Además, el empleo de animales permite analizar la efectividad de algunos fármacos, como el antioxidante $\mathrm{N}$ acetilcisteina, en la prevención de dichas alteraciones comprobando si restan o no eficacia al tratamiento antineoplásico. La valoración aguda o a muy corto plazo de estos trabajos puede considerarse como una limitación a superar. Pero, al evaluar el efecto a 8 meses vista desde el final del tratamiento, también se han encontrado daños sutiles aunque prolongados en la realización de las tareas propuestas, por ejemplo, perjuicios en memoria espacial e inhibición del comportamiento ${ }^{(41)}$.

Otros trabajos con animales se complementan con experimentos in vitro, los resultados arrojan una alta correspondencia de los resultados in vivo e in vitro, estos últimos pueden ser una herramienta útil, tanto para identificar la toxicidad asociada a diferentes esquemas quimioterapéuticos como para desarrollar una protección selectiva de las células no tumorales ${ }^{(42)}$. Otra ventaja de los estudios in vitro es que se convierten en una alternativa para reducir el daño causado a animales de laboratorio.

\section{Instrumentos y estrategias de evaluación del rendimiento cognitivo}

Los test neuropsicológicos clásicos han recibido diversas críticas en trabajos precedentes, como son el excesivo tiempo requerido para la administración de algunas baterías neuropsicológicas, la escasa validez ecológica de ciertas pruebas, la alta sensibilidad al efecto de la práctica y el aprendizaje (como pudiera ser el caso del High Sensitivity Cognitive Screen $\left.{ }^{(43)}\right)$, así como la escasa sensibilidad a deterioro sutil. Esto no quiere decir que los test clásicos no sean útiles para el estudio del chemobrain, sino que deben ser correctamente seleccionados, tanto por su fiabilidad y validez, como por su adecuación a las características de la población a la que van destinados, sin olvidar que se trata de personas inmersas en un proceso de curación de una enfermedad grave.

Actualmente, se está fomentando la obtención de datos mediante técnicas de neuroimagen y técnicas psicofisiológicas. La resonancia magnética se ha empleado en varios trabajos, de caso único ${ }^{(44)}$, estudio comparativo entre hermanas geme$\operatorname{las}^{(45)}$ y entre grupos. Algunos datos sostienen que el deterioro cognitivo detectado por las imágenes de resonancia magnética se correlacionan con el deterioro cognitivo percibido pero no con los resultados de los test clásicos ${ }^{(46)}$; también se objetiva más hipertensión en la sustancia blanca, y mayor extensión de actividad cerebral en la realización de una tarea de memoria ${ }^{(45)}$ y menor sustancia gris y blanca en los giros prefrontal, parahipocampal y cingulado en 
las mujeres que sufrieron cáncer de mama tratadas con quimioterapia ${ }^{(47)}$. De igual forma, mediante resonancia magnética se han encontrado daños en la actividad del córtex frontal, el cerebelo y los ganglios basales en mujeres de cáncer de mama que habían recibido quimioterapia hacía 5-10 años ${ }^{(48)}$; la quimioterapia puede afectar a la sustancia blanca del cuerpo callo$\mathrm{so}^{(49)}$ y a la sustancia gris cortical(50).

Mediante encefalograma, se observan alteraciones electrofisiológicas en los potenciales P3 en personas con cáncer de mama tratadas con dosis altas de quimioterapia hacía 4 años ${ }^{(51)}$. En otro trabajo, que contó con la participación de 53 pacientes de cáncer de mama tratadas con diferentes tipos de quimioterapia y 23 con la enfermedad en estadio I no tratadas con quimioterapia, se registraron reducciones en la amplitud de P3 y se constató que algunos esquemas pueden alterar también su latencia(52).

Como puede apreciarse, el empleo de diferentes instrumentos de evaluación puede enriquecer el conocimiento de este efecto secundario.

\section{Mecanismos subyacentes al déficit cognitivo asociado a la quimioterapia}

Tanto los estudios con humanos como con animales están siendo de gran utilidad para dilucidar los mecanismos que subyacen al chemobrain. Sobre estos, Ahles y Saykin ${ }^{(53)}$, realizan un análisis pormenorizado, repasando algunos factores que podrían inducir o modular los cambios cognitivos asociados al uso de quimioterapia. Los autores subrayan que la genética del individuo tiene un peso importante en el desarrollo y evolución del chemofog. Las diferencias individuales en cuanto a la vulnerabilidad previa de cada paciente, debida al polimorfismo genético humano, pueden incrementar el riesgo a padecer enfermedades como el cáncer y el dete- rioro cognitivo. La confluencia de estos factores eleva el riesgo de sufrir deterioro a largo plazo. Algunos de los posibles factores involucrados que participan en el desarrollo de esta toxicidad serían:

- En primer lugar, pequeñas cantidades de los citostáticos podrían atravesar la barrera hematoencefálica, aumentando la muerte celular y disminuyendo la división celular en zonas subventriculares (cantidades insuficientes para abordar eficazmente un tumor cerebral, podrían producir deterioro cognitivo); en este sentido, las personas con una genética que haga más permeable su barrera hematoencefálica serán más sensibles a problemas cerebrales.

- Daño en el ADN, cuanto mayor sea éste y menor sea la eficiencia de los mecanismos para su reparación, más probable sería el deterioro neuropsicológico tanto antes como después de la quimioterapia; por su parte, la quimioterapia disminuye la capacidad antioxidante de las células y con ello incrementa el daño del ADN.

- El papel de las citoquinas en el deterioro cognitivo. Igualmente, los pacientes con una genética más vulnerable a un mal funcionamiento de las citoquinas (uno o más alelos asociados a la disregulación de éstas) haría más probable sufrir problemas cognitivos, antes y después de la quimioterapia.

- Serían más vulnerables al deterioro cognitivo las personas cuyos genes posean alelos asociados a la disregulación de los telómeros, a la reparación neuronal (por ejemplo, el alelo APOE E4) o al decremento de la actividad de los neurotransmisores (por ejemplo la catecol-O-metiltransferasa).

- La quimioterapia altera los niveles de testosterona y estrógenos, consideradas hormonas neuroprotectoras. En la menopausia, la reducción normal de estrógenos perjudica el rendimiento cognitivo, sobre todo la memoria verbal. La quimioterapia puede provocar una menopausia inducida 
e interferir en la secreción hormonal, facilitando los problemas cognitivos.

Por su parte, Vardy resume en cinco puntos los posibles mecanismos inherentes a la quimioterapia que le confieren su capacidad de alterar el funcionamiento $\operatorname{cognitivo~}^{(3)}$ :

- Efectos neurotóxicos directos.

- Daño oxidativo.

- Cambios hormonales inducidos.

- Desregulación del sistema inmune a través de la liberación de citoquinas.

- Coagulación de la sangre en pequeños vasos del sistema nervioso central.

\section{Estudios sobre el tratamiento del daño cognitivo quimio-inducido}

Otro punto de interés es nuestro análisis es la valoración del afrontamiento terapéutico del daño cognitivo quimioinducido. Son varios los trabajos realizados en muestras de mujeres de cáncer de mama que emplean fármacos para valorar su eficacia en la mejora del rendimiento cognitivo (ver tabla 2):

- Epoetina-alfa: presenta algunos resultados confusos. Mientras que en un estudio parece mitigar la fatiga y mejorar el rendimiento cognitivo que sigue al tratamiento en personas con cáncer de mama $^{(54) ;}$ en otro trabajo no se aprecian diferencias significativas ${ }^{(5)}$.

- Eritropoyetina: algunos datos indican la posibilidad de una mejoría del rendimiento cognitivo, evaluada mediante el Mini Mental State Examination, cuando la anemia de los pacientes es tratada mediante este fármaco ${ }^{(56)}$.

- Modafinilo: se han hallado incrementos en memoria y atención tras su administración ${ }^{(57)}$.

- D-Metilfenidato: este fármaco se ha mostrado inefectivo ${ }^{(58)}$.

Los efectos positivos de ciertos fárma$\cos$ se han examinado mediante experimentos con ratas de laboratorio:
- $\gamma$-glutamil-cisteina-etil-ester: muestran un efecto protector del estrés oxidativo producido por inyecciones previas de adriamicina $^{(59)}$.

- Fluoxetina (Inhibidor Selectivo de la Recaptación de Serotonina): parece mejorar la proliferación celular del hipocampo y la memoria de trabajo que fueron alteradas previamente por el 5 -fluorouracilo ${ }^{(60)}$.

Además, se han realizado estudios empleando métodos no farmacológicos para el control del "chemobrain".

- "Entrenamiento en adaptación de memoria y atención" (MAAT por sus siglas en inglés) breve y fácil de aplicar, cuyos efectos en mujeres con cáncer de mama se constatan hasta seis meses después de finalizar el entrenamiento, y capaz de mejorar no sólo las quejas sino también el rendimiento en los test y la satisfacción del paciente por el control de sus síntomas $\operatorname{cognitivos}^{(61)}$. Mediante este entrenamiento se produce una mejoría de la memoria verbal y la calidad de vida ${ }^{(62)}$.

- Meditación: puede ser una herramienta útil tanto para conseguir una sensación subjetiva de mejoría como para reducir objetivamente el déficit cognitivo, favoreciendo la atención y el control mental, teniendo además otras ventajas para la salud al facilitar estados de relajación y bienestar ${ }^{(63)}$.

- Por otro lado, Poppelreuter, Weis y Bartsch, en una muestra de mujeres con cáncer de mama, compararon dos tipos de intervenciones neuropsicológicas frente a subgrupo sin entrenamiento, sin llegar a identificar efectos específicos de la intervención, y destacando la permanencia de un subgrupo de afectados seis meses después del entrenamiento ${ }^{(64)}$.

Los resultados de los diferentes trabajos de investigación aún son dispares. En este sentido, Vardy, Rourke y Tannock, nos recuerdan las dificultades que supone la falta de consistencia metodológica en los estudios. Cada investigación adopta diseños 


\section{Tabla 2. Estudios que abordan el tratamiento del déficit cognitivo asociado a la quimioterapia}

\begin{tabular}{|c|c|c|}
\hline \multicolumn{3}{|c|}{ Resultados alentadores } \\
\hline \multirow{5}{*}{ Fármacológicos } & \multirow{3}{*}{$\begin{array}{l}\text { Mujeres cáncer } \\
\text { de mama }\end{array}$} & Epoetín alfa \\
\hline & & Eritropoyetina \\
\hline & & Modafinilo \\
\hline & \multirow{2}{*}{ Roedores } & Ganma-glutamil-cisteina-etil-éster \\
\hline & & ISSR \\
\hline $\begin{array}{l}\text { Entrenamiento en Adapta- } \\
\text { ción de Memoria y Aten- } \\
\text { ción }\end{array}$ & $\begin{array}{l}\text { Mujeres cáncer } \\
\text { de mama }\end{array}$ & Entrenamiento cognitivo-conductual \\
\hline Meditación & $\begin{array}{l}\text { Pacientes onco- } \\
\text { lógicos }\end{array}$ & \\
\hline \multicolumn{3}{|c|}{ No efectivos } \\
\hline \multirow[t]{2}{*}{ Farmacológico } & $\begin{array}{l}\text { Mujeres cáncer } \\
\text { de mama }\end{array}$ & D-metilfenidato \\
\hline & Tumores sólidos & Epoetín alfa \\
\hline $\begin{array}{l}\text { Entrenamiento neusopsico- } \\
\text { lógico }\end{array}$ & $\begin{array}{l}\text { Mujeres cáncer } \\
\text { de mama }\end{array}$ & \\
\hline \multicolumn{3}{|c|}{ Bajo estudio/propuesta } \\
\hline Ginkgo Biloba & & \\
\hline
\end{tabular}

y definición de deterioro cognitivo diferentes ${ }^{(65)}$. Miden diversos dominios empleando multitud de pruebas de evaluación que en ocasiones divergen extraordinariamente (desde screening rudimentarios como el Mini Mental State Examination hasta baterías como la computerizada (ANTAB). Y esto, aunque proporcione un mosaico de resultados que en su mayoría convergen en un mismo punto, dificulta la tarea de comparación de estudios. Por todo ello, se hace necesario utilizar métodos estadísticos estándares y realizar un esfuerzo de colaboración para homogeneizar los métodos para la evaluación ${ }^{(66)}$.

La puesta en marcha de diferentes workshops internacionales, es una muestra del esfuerzo y el interés de la comunidad científica por superar las limitaciones y hacer una puesta en común de las aportaciones que evidencian cambios cognitivos por el uso de quimioterapia. Estos encuentros congregan a profesionales de la neuropsicología, psicología clínica y experimental, y de oncología, entre otros. Los expertos allí reunidos presentan los últimos estudios, los avances y las dificultades, y tratan temas de interés relacionados (colaboración, orientaciones para el futuro, divulgación de información). Junto a estos paneles, se ha constituido el International Cognition and Cancer Task Force (ICCTF). El primer workshop se realizó en Canadá en 2003, del que se subrayan cuatro grandes bloques: alcance del problema; problemas de la evaluación cognitiva; 
posibles mecanismos y prevención; tratamiento y rehabilitación, donde se resalta la necesidad de estudios longitudinales prospectivos con comparaciones grupales y control estadístico del efecto de la práctica ${ }^{(67)}$. En el segundo, en Venecia en 2006, se puso de manifiesto que, mientras unos estudios defienden la naturaleza difusa de este deterioro, la evaluación neuropsicológica sugiere un deterioro frontal, que afectaría a la velocidad de procesamiento de la información, la atención, la evocación de recuerdos y la función ejecutiva. También hacen constar la necesidad de establecer guías para orientar las futuras investigaciones y aportar información sobre cómo abordar los síntomas ${ }^{(68)}$.

\section{CONCLUSIÓN}

Como resultado de la búsqueda bibliográfica emprendida y el análisis de la información, podemos decir que el estudio de la naturaleza y alcance del impacto cognitivo asociado a la quimioterapia es un tema de vigente actualidad. Algunas personas enfermas de cáncer pueden sufrir daño cognitivo asociado al tratamiento con quimioterapia. Este deterioro suele ser sutil y tiende a desaparecer con el tiempo, pero en algunos casos puede convertirse en una grave secuela.

Muchos de los estudios realizados se han centrado en mujeres con cáncer de mama. Sin embargo, el daño no se restringe a esta población, sino que parece afectar a otros enfermos de cáncer, como pueden ser los afectados de cáncer testicular, ovárico, colorrectal y pulmonar entre otros.

Los estudios con animales de laboratorio, así como el empleo de técnicas de neuroimagen y electrofisiológicas están permitiendo un conocimiento más profundo de los mecanismos de acción que están en la base de éste fenómeno. Algunos de los estudios con ratas permiten valorar la toxicidad de determinados fármacos en particular y realizar experimentos que serían éticamente cuestionables en seres humanos. Las técnicas de neuroimagen están corroborando daños cerebrales en la materia gris y blanca de los sujetos tratados con agentes quimioterapéuticos. Los datos ponen de manifiesto una mayor activación cerebral en determinadas tareas de aprendizaje y memoria, lo cual indicaría una tendencia a la compensación de los daños y, por consiguiente, explicaría la progresiva recuperación que se observa en los pacientes con el paso del tiempo.

Tanto las variables psico-sociales (como la edad, nivel de estudios, apoyo social percibido) la vulnerabilidad genética de partida (por ejemplo, la permeabilidad de la barrera hematoencefálica, la presencia alelos asociados a la disregulación de los telómeros) como aquellos inherentes al tratamiento (tales como la neurotoxicidad, estrés oxidativo, inducción de cambios hormonales) hacen que este fenómeno presente una manifestación única en cada individuo.

En los últimos años se hace más patente el interés por realizar trabajos de prevención y/o rehabilitación del deterioro asociado a la quimioterapia. Nuevamente, los estudios con animales resultan eficaces para valorar la influencia de determinados fármacos en el rendimiento y su compatibilidad con los tratamientos sistémicos. Al parecer, tanto las intervenciones farmacológicas como el entrenamiento cognitivoconductual pueden reportar beneficios.

Los hallazgos recientes son alentadores, porque permiten la toma de consciencia de un efecto secundario de la quimioterapia que puede afectar gravemente a la vida de las personas, y porque ya se están poniendo en marcha instrumentos para su control. No obstante, los datos siguen siendo aún imprecisos y, en ocasiones, incongruentes. Por lo que ratificamos la imperiosa necesidad de establecer guías de 
investigación que tengan presentes estas consideraciones.

La situación actual del panorama nacional en la evaluación de los efectos de la quimioterapia en las funciones cognitivas es escasa e insuficiente. Sería muy positivo que se incentivara el estudio del chemobrain en nuestro país, para conseguir un conocimiento adecuado del mismo, permitir su prevención y control y con ello potenciar el bienestar y preservar la calidad de vida de las personas afectadas.

\section{REFERENCIAS BIBLIOGRÁFICAS}

1. Rubio B, Sirgo A, Forcadell E, Mele M, Guma J. Deterioro cognitivo inducido por los tratamientos oncológicos sistémicos en el cáncer de mama no metastático: revisión de estudios. Psicooncología 2009; 6: 83120.

2. López S, Cruzado JA, Feliu J. Rendimiento cognitivo, estado emocional y calidad de vida en pacientes de cáncer de colon previamente a recibir tratamiento de quimioterapia. Psicooncología 2009; 6: 121-37.

3. Vardy J. Neurocognitive effects of chemotherapy in adults. Aust Prescr 2008; 31: 22-24.

4. Argyriou A, Assimakopoulos K, Iconomou G, Giannakopoulou F, Kalofonos H. Either caller "chemobrain" or "chemofog", the long-term chemotherapy-induced cognitive decline in cancer survivors is real. J Pain Symptom Manage 2010 : 41 : 126-39. Doi : http://dx.doi.org/10.1016/j.jpainsymman.2010.04.021

5. Hess LM, Insel KC. Chemotherapy-related change in cognitive function: a conceptual model. Oncol Nurs Forum 2007; 34: 981 94. Doi:10.1188/07.ONF.981-994

6. Rodin MB, Wallace JA, Lacy M, Kuball K, Pykkonen B, Freming G. Does adjuvant chemotherapy (CT) exacerbate cognitive impairment in elderly breast cancer (BC) patients? Results of a prospective, longi- tudinal study. J Clin Oncol 2006; 24 (20 Supplement): 10530.

7. Vardy J, Wong K, Yi Q, Park A, Maruff $P$, Wagner $L$ et al. Assessing cognitive function in cancer patients. Support Care Cancer 2006; 14: 1111-18. Doi:10.1007/ s00520-006-0037-6

8. Wefel JS, Saleeba AK, Buzdar AU, Meyers CA. Acute and late onset cognitive dysfunction associated with chemotherapy in women with breast cancer. Cancer 2010; 116: 3348-56. Doi:10.1002/cncr.25098

9. Hurria A, Somlo G, Ahles T. Renaming "chemobrain". Cancer Invest 2007; 23: 373-7. Doi:10.1080/07357900701506672

10. Bender CM, Sereika SM, Berga SL, Vogel VG, Brufsky AM, Paraska KK et al. Cognitive impairment associated with adjuvant therapy in breast cancer. Psychooncology 2006; 15: 422-30. Doi:10.1002/pon.964

11. Collins B, Mackenzie J, Stewart A, Bielajew C, Verma S. Cognitive effects of chemotherapy in post-menopausal breast cancer patients 1 year after treatment. Psychooncology 2009; 18: 134-43. Doi:10.1002/ pon. 1379

12. Correa DD, Ahles TA. Cognitive adverse effects of chemotherapy in breast cancer patients. Cur Opin Support Palliat Cancer 2007; 1: 57-62. Doi:10.1097/ SPC.0b013e32813a328f

13. Wagner LI, Sweet J, Butt Z, Beaumont J, Havlin KA, Sabatino $T$ et al. Trajectory of cognitive impairment during breast cancer treatment: a prospective analysis. J Clin Oncol, 2006; 24 (20 Suppl): 8500.

14. Jansen CE, Dodd M, Miaskowski CA, Dowling GA, Kramer J. Preliminary results of a longitudinal study of changes in cognitive function in breast cancer patients undergoing chemotherapy with doxorubicin and cyclophosphamide. Psychooncology, 2008; 17: 1189-95. Doi:10.1002/pon.1342

15. Ahles TA, Saykin AJ, McDonald BC, Li Y, Furstenberg CT, Hanscom BS et al. Longitudinal assessment of cognitive changes associated with adjuvant treatment for 
breast cancer: impact of age and cognitive reserve. J Clin Oncol 2010; 28: 4434-40. Doi:10.1200/JCO.2009.27.0827

16. Schagen SB, Muller MJ, Boogerd W, MeIlenbergh GJ, van Dam FSAM. Change in cognitive function after chemotherapy: A prospective longitudinal study in breast cancer patients. J Natl Cancer Inst 2006; 98: 1742-45. Doi:10.1093/jnci/djj470

17. Stewart A, Collins B, Mackenzie J, Tomiak E, Verma S, Bielajew C. The cognitive effects of adjuvant chemotherapy in early stage breast cancer: a prospective study. Psychooncology 2008; 17: 122-30. Doi:10:1002/pon.1210

18. Quesnel C, Savard J, Ivers H. Cognitive impairments associated with breast cancer treatments: results from a longitudinal study. Breast Cancer Res Treat 2009; 116: 113-23. Doi:10.1007/s10549-008-0114-2

19. Ahles TA, Saykin AJ, McDonald BC, Furstenberg CT, Cole BF y Hanscom BS. Cognitive function in breast cancer patients prior to adjuvant treatment. Breast Cancer Res Treat 2008; 110: 143-52. Doi:10.1007/ s10549-007-9686-5

20. Hurria A, Goldfarb S, Rosen C, Holland J, Zuckerman E, Lachs MS et al. Effect of adjuvant breast cancer chemotherapy on cognitive function from the older patient's perspective. Breast Cancer Res Treat 2006; 98: 343-8. Doi:10.1007/s10549-006-9171-6

21. Shilling V, Jenkins V. Self-reported cognitive problems in women receiving adjuvant therapy for breast cancer. Eur J Oncol Nurs 2007; 11: 6-15. Acceso el 12 de Agosto, 2010. Disponible en: http://www.ncbi.nlm. nih.gov/pubmed/16844416 Doi:10.1016/j. ejon.2006.02.005

22. Jenkins $V$, Shilling V, Deustsch G, Bloomfield D, Morris R, Allan S et al. A 3-year prospective study of the effects of adjuvant treatments on cognition in women with early stage breast cancer. Br J Cancer 2006; 94: 828-34. Doi.10.1030/sj.bjc.6603029

23. Mehlsen M, Pedersen AD, Jensen AB, Zachariae R. No indications of cognitive side- effects in a prospective study of breast cancer patients receiving adjuvant chemotherapy. Psychooncology 2009; 18, 248-57. Doi:10.1002/pon.1398

24. Tager FA, Mckinley PS, Schnabel FR, ElTamer M, Cheung YKK, Fang $Y$ et al. The cognitive effects of chemotherapy in postmenopausal breast cancer patients: a controlled longitudinal study. Breast Cancer Res Treat 2010; 123: 25-34. Doi:10.1007/ s10549-009-0684-7

25. Eberhardt B, Dilger S, Musial F, Wedding $U$, Weiss T, Miltner WHR. Short-term monitoring of cognitive functions before and during the first course of treatment [Abstract]. J Cancer Res Clin Oncol 2006; 132: 234-40. Doi:10.1007/s00432-005-0070-8

26. Eberhardt B, Dilger S, Musial F, Wedding $U$, Weiss T, Miltner WHR. Medium-term effects of chemotherapy in older cancer patients. Support Care Cancer 2006; 14: 216-22. Doi:10.1007/s00520-005-0894-4

27. Mancuso A, Migliorino M, De Santis S, Saponiero A, De Marinis F. Correlation between anemia and fuctional/cognitive capacity in elderly lung cancer patients treated with chemotherapy. Ann Oncol 2006; 17:146-50. Doi:10.1093/annonc/mdj038

28. Vardy J, Rourke S, Pond GR, Galica J, Park A, Dhillon $\mathrm{H}$, et al. Cognitive function and fatigue in cancer patients after chemotherapy: a longitudinal cohort study in patients with colorectal cancer (CRC). J Clin Oncol 2007: 25 (Meeting Abstracts) (18): Suppl 9099

29. Vardy JL, Xu W, Booth CM, Park A, Dodd A, Rourke $S$ et al. Relation between perceived cognitive function and neuropsychological performance in survivors of breast and colorectal cancer. J Clin Oncol 2008; 26 (Meeting Abstracts) (15): Suppl 9520

30. Vardy J, Dhillon H, Xu W, Dodd A, Park A, Rourke SB, et al. Cognitive function and fatigue in colorectal cancer (CRC) patients: Baseline assessments prior to chemotherapy. J Clin Oncol 2009; 27 (Meeting Abstracts) (15): Suppl 9557 
31. Schagen SB, Boogerd W, Muller MJ, Huinink WT, Moonen L, Meinhardt W et al. Cognitive complaints and cognitive impairment foIlowing BEP chemotherapy in patients with testicular cancer. Acta Oncol 2008; 47: 6370. Doi:10.1080/02841860701518058

32. Wefel, Vidrine, Veramonti, Meyers, Marani, Hoekstra et al. Cognitive impairment in men with testicular cancer prior to adjuvant therapy. Cancer 2011; 117: 190-6. Doi.10.1002/cncr.25298

33. Myers JS, Sousa VD y Donovan HS. Predictors of self-reported memory problems in patients with ovarian cancer who have received chemotherapy. Oncol Nurs Forum 2010; 35: 596-603. Doi:10.1188/10. ONF.596-603

34. Winocur G, Vardy J, Binns MA, Kerr L y Tannock I. The effects of the anti-cancer drugs, methotrexate and 5-fluorouracil, on cognitive function in mice [Abstract]. Pharmacol Biochem Behav, 2006; 85: 65-75. Doi:10.1016/j.pbb.2006.07.010

35. Lee GD, Longo DL, Wang Y, Rifkind JM, Abdul-Raman L, Mamczarz JA et al. Transient improvement in cognitive function and synaptic plasticity in rats following cancer chemotherapy. Clin Cancer Res 2006; 12: 198-205. Doi:10.1158/10780432.CCR-05-1286

36. Reiriz AB, Reolon GK, Preissles T, Rosado $\mathrm{JO}$, Henriques JAP, Roesler R et al. Letter to the Editor: Cancer chemotherapy and cognitive function in rodent models: memory impairment induced by cyclophosphamide in mice. Clin Cancer Res 2006; 12: 5000-1.

37. Tangpong J, Cole MP, Sultana R, Estus S, Vore $M$, St. Clair W et al. Adriamycin-mediated nitration of manganese superoxide dismutase in the central nervous system: Insight into the mechanism of chemobrain. J Neurochem 2007; 100: 191-201. Doi:10.1111/j.1471-4159.2006.04179.x

38. Konat GW, Kraszpulski M, James I, Zhang H-T y Abraham J. Cognitive dysfunction induced by chronic administration of common cancer chemotherapeutics in rats. Metab Brain Dis 2008; 23: 325-33. Doi:10.1007/11011-008-9100-y

39. Gandul MJ, Ehrlichman RS, Rudnick N, Siegel SJ. A novel electrophysiological model of chemotherapy-induced cognitive impairments in mice. Neuroscience 2008; 157: 95-104. Doi:10.1016/j.neuroscience.2008.08.060

40. Foley JJ, Raffa RB, Walter EA. Effects of chemotherapeutic agents 5-fluorouracil and methotrexate alone and combined in a mouse model of learning and memory. Psychopharmacology 2008; 199: 527-38. Doi:10.1007/s00213-008-1175-y

41. Fardell JE, Vardy J, Logge W, Johnston I. Single high dose treatment with methotrexate causes long-lasting cognitive dysfunction in laboratory rodents. Pharmacol, Bioschem Behav 2010; 97: 333-39. Doi:10.1016/j. pbb.2010.08.019

42. Dietrich J, Han R, Yang Y, Mayer-Pröschel M, Noble M. CNS progenitor cells and aligodendrocytes are targets of chemotherapeutic agents in vitro and in vivo. J Biol, 2006; J Biol. 2006; 5: 22.1-22. [En línea] [Acceso el 12 de noviembre, 2008]. Disponible en: http://jbiol.com/content/pdf/ jbiol50.pdf

43. Schagen SB, Dam FS, van Dam Does cognitive impairment after chemotherapy for breast cancer improve over time or does practice make perfect? J Clin Oncol 2006; 24: 51701. Doi:10.1200/JCO.2006.07.8303

44. Swayampakula AK, Alkhouri N, Haut MW, Abraham J. Cognitive impairment with significant brain parenchymal volume loss following standard adjuvant chemotherapy in a patient with breast cancer. Clin Adv Hematol Oncol 2007; 5: 985-7.

45. Ferguson RJ, McDonald BC, Saykin AJ, Ahles TA. Brain structure and function differences in monozygotic twins: Possible effects of breast cancer chemotherapy. J Clin Oncol 2007; 25: 3866-70. Doi:10.1200/JCO.2007.10.8639

46. Booth CM, Vardy J, Crawley A, Rourke S, Pond G, Wagner L, et al. Cognitive impair- 
ment associated with chemotherapy for breast cancer: an exploratory case-control study [Abstract]. J Clin Oncol 2006; 24 (18 Suppl): 8501.

47. Inagaki M, Yoshikawa E, Matsuoka Y, Sugawara $Y$, Nakano T, Akechi T, et al. Smaller regional volumes of brain gray and white matter demonstrated in breast cancer survivors exposed to adjuvant chemotherapy. Cancer 2007; 109: 146-56. Doi:10.1002/ cncr.22368

48. Silverman CJ, Steven CJ, Castellon SA, Lai J, Pio BS, Abraham L, et al. Altered frontocortical, cerebellar, and basal ganglia activity in adjuvant-treated breast cancer survivors 5-10 years after chemotherapy. Breast Cancer Res Treat 2007; 103, 30311. Doi:10.1007/s10549-006-9380-z

49. Abraham J, Aut, MW, Moran MT, Filburn $\mathrm{S}$, Lemiuex S. Kuwabara H. Adjuvant chemotherapy for breast cancer: effects on cerebral white matter seen in diffusion tensor imaging. Clin Breast Cancer 2008; 8: 8891. Doi:10.3816/CBC.2008.n.007

50. McDonald BC, Conroy SK, Ahles T, West JD, Saykin AJ. Gray matter reduction associated with systemic chemotherapy for breast cancer: A Prospective MRI study [Abstract]. Breast Cancer Res Treat 2010; 123: 819-28. Doi:10.1007/s10549-010-1088-4

51. Kreukels VPC, Schagen SB, Ridderinkhof KR, Boogerd W, Hamburger HL, Muller MJ et al. Effects of high-dose and conventionaldose adjuvant chemotherapy on long-term cognitive sequelae in patients with breast cancer: an electrophysiologic study. Clin Breast Cancer 2006; 7: 67-8. Doi:10.3816/ CBC.2006.n.015

52. Kreukels BPC, Hamburger HL, Ruiter MB, van Dam FSAM, Ridderinkhof KR, Boogerd $W$, et al. ERP amplitude and latency in breast cancer survivors treated with adjuvant chemotherapy. Clin Neurophysiol 2008; 119: 533-41. Doi:10.1016/j. clinph.2007.11.011

53. Ahles T, Saykin A. Candidate mechanisms for chemotherapy-induced cognitive chan- ges. Nat Rev Cancer 2007; 7: 192-201. Doi:10.1038/nrc2073

54. O'Shaughnessy JA, Vukelja SJ, Holmes FA, Savin, M, Jones $M$, Royall D et al. Feasibility of quantifying the effects of epoetin alfa therapy on cognitive function in women with breast cancer undergoing adjuvant or neoadjuvant chemotherapy. Clin Breast Cancer 2005; 5: 439-46. Doi:10.3816/ CBC.2005.n.002

55. Iconomou G, Koutras A, Karaivazoglou K, Kalliolias GD, Assimakopoulos K, Argyriou AA et al. Effect of epoetin alpha therapy on cognitive function in anaemic patients with solid tumours undergoing chemotherapy. Eur J Cancer Care 2008; 17: 535-41. Doi:10.1111/j.1365-2354.2007.00857.x

56. Massa E, Madeddu C, Lusso MR, Gramignano G, Mantovani G. Evaluation of the effectiveness of treatment with erythropoietin on anemia, cognitive functioning and functions studied by comprehensive geriatric assessment in elderly cancer patients with anemia related to cancer chemotherapy. Crit Rev Oncol Hematol 2006; 57: 17582. Doi.10.1016/j.critrevonc.2005.06.001

57. Kohli S, Fisher SG, Tra Y, Adams MJ, Mapstone MG, Wesnes KA et al. The effect of modafinil on cognitive function in breast cancer survivors. Cancer 2009; 115: 260516. Doi:10.1002/cncr.24287

58. Mar Fan HG, Clemons M, Xu W, Chemerynsky I, Breunis H, Braganza S, et al. A randomised, placebo-controlled, double-bling trial of the effects of d-methylphenidate on fatigue and cognitive dysfunction in women undergoing adjuvant chemotherapy for breast cancer. Support Care Cancer 2008; 16: 364-71. Doi:10.1007/s00520-007-0341-9

59. Joshi G, Hardas S, Sultana R, St. Clair DK, Vore $M$, Butterfield DA. Glutathione elevation by $\boldsymbol{\gamma}$-glutamyl cysteine ethyl ester as a potential therapeutic strategy for preventing oxidative strees in brain mediated by in vivo administration of adriamycin: Implication for chemobrain. J Neurosci Res 2007; 85: 497-503. Do1: 10.1002/jnr.21158 
60. Mustafa S, Umka J, Lyons L, Salman A, Chur-yoe GT, Bhalla $\mathrm{N}$ et al. Fluoxetine improves the memory deficits caused by the chemotherapy agent 5-fluorouracil. Behav Brain Res 2010; 208: 112-17. Doi:10.1016/j.bbr.2009.11.017

61. Ferguson RJ, Ahles TA, Saykin AJ, MCDonald BC, Furstenberg, CT, Cole BF et al. Cognitive-behavioural management of chemotherapy-related cognitive change. Psychooncology 2007; 16: 772-777. Doi:10.1002/pon.1133

62. Ferguson RJ, McDonald BC, Rocque MA, Furstenberg CT, Horrigan S, Ahles TA, Saykin AJ. Development of CTB for chemotherapy-related cognitive change: results of a waitlist control trial. Psychooncology 2010. Doi:10.1002/pon.1878

63. Biegler KA, Chaoul MA, Cohen L. Cancer, cognitive impairment, and meditation. Acta Oncol 2009; 48: 18-26. Doi:10.1080/02841860802415535

64. Poppelreuter M, Weis J, Bartsch HH. Effects of specific neuropsychological training programs for breast cancer patients after adjuvant chemotherapy. J Psychosoc Oncol 2009; 2: 274-96. Doi:10.1080/07347330902776044

65. Vardy J, Rourke S, Tannock IF. Evaluation of cognitive function associated with chemotherapy: A review of published studies and recommendations for future research. J Clin Oncol 2007; 25: 2455-63. Doi:10.1200/2006.08.1604

66. Shilling V, Jenkins V, Trapala IS. The (mis) classification of chemo-fog-methodological inconsistencies in the investigation of cognitive impairment after chemotherapy. Breast Cancer Res Treat 2006; 95: 125-9. Doi:10.1007/s10549-005-9055-1

67. Tannock IF, Ahles TA, Ganz PA, van Dam FS. Cognitive impairment associated with chemotherapy for cancer: Report of a Workshop. J Clin Oncol 2004; 22: 2233-9. Doi:10.1200/JCO.2004.08.094

68. Vardy J, Wefel JS, Ahles T, Tannock IF, Schagen SB. Cancer and cancer-therapy related cognitive dysfunction: an international perspective from the Venice cognitive workshop. Ann Oncol 2008; 19: 623-29 Doi:10.1093/annonc/mdm500 